
\title{
25 Research Square \\ Formation of Metal Soaps In Oil Paintings Under Variable Environmental Conditions
}

\section{Piovesan}

Complutense University of Madrid Faculty of Fine Arts: Universidad Complutense de Madrid Facultad de Bellas Artes

Marta Pérez-Estébanez ( $\nabla$ mperezes@ucm.es )

Complutense University of Madrid https://orcid.org/0000-0002-1525-3962

\section{San Andrés-Moya}

Complutense University of Madrid Faculty of Fine Arts: Universidad Complutense de Madrid Facultad de Bellas Artes

\section{Research article}

Keywords: Metal soap, Oil paintings, Environmental conditions, ATR-FTIR, Conservation of cultural heritage

Posted Date: July 7th, 2021

DOl: https://doi.org/10.21203/rs.3.rs-667290/v1

License: (c) (1) This work is licensed under a Creative Commons Attribution 4.0 International License. Read Full License 


\section{Abstract}

Formation of metal soaps in model paintings under cyclic temperature and relative humidity conditions has been studied by ATR-FTIR and compared with the standard controlled conditions generally maintained in many museums and storerooms. Three different white pigments (lead, zinc and titanium white) and two types of linseed oils (cold-pressed and alkali-refined) have been tested. The behaviour in short times (up to 6 weeks) depends on the pigment and environmental conditions. The relative humidity conditions have been shown to influence the metal soap formation in more degree than the temperature. Ageing under low cyclic relative humidity (30-50\%) promotes metal soap formation in both lead and zinc white pigments, compared to high relative humidity (50-90\%) conditions. Besides, the formation of zinc soaps continues for 6 weeks when low cyclic relative humidity is used, but it stops after 3 weeks under the other ageing conditions studied.

\section{Introduction}

In the second half of the 90 s, some of the damage phenomena produced in oil paintings were associated, for the first time, with the formation of metal soaps. Since then, the harmful effect of these compounds on the structural integrity and aesthetical appearance of works of art is well known $[1,2]$.

Metal soaps are metal carboxylates containing both fatty acids and metal cations. In traditional oil paintings, metal soaps are formed by the chemical reaction between the fatty acids of the drying oil with the metal ions $(\mathrm{Zn}, \mathrm{Pb}, \mathrm{Cu}$, etc) of some pigments or additives. In modern paintings, these compounds are sometimes added on purpose as dispersion agents, stabilizers, and extenders [3]. Metal soaps show up in the painting in a heterogeneous way, causing different kinds of damages: protrusions, efflorescence, increased transparency, etc. [4-7].

The damage phenomena due to metal soaps have been pointed out in paintings of different age, since Rembrandt (S. XVII) to Salvador Dalí (S. XX), evidencing the extension of the problem and alarming the conservation departments of many museums all over the world. It is, indeed, a problem intrinsic to the painting components, unavoidable though, in some kinds of artworks. Although some metal soap aggregations have been successfully reduced using cleaning treatments [8], great caution is needed in the choice and use of cleaning agents. Furthermore, when the damage compromises the structural integrity of the paint layers, no restoration treatment exists that can return the paintings to their original condition. Preventive conservation is, therefore, the only strategy to adopt.

Although the formation mechanism of metal soaps has been extensively studied, the correlation with the environmental conditions of conservation still needs a deeper investigation. Some studies have demonstrated that certain conditions of temperature and relative humidity favour the formation of metal soaps and their subsequent crystallization [9-15]. However, in most of the published works, extreme conditions of ageing were used, which are far away from the real conservation environments. Besides, the normal fluctuations of the environmental parameters with time, were not taken into account. 
Hence, this research was born to study the formation of metal soaps in environmental conditions that are more similar to the real ones, trying to simulate both control and uncontrol storage rooms, with the aim of establishing the best values of temperature and relative humidity to avoid or delay the formation of metal soaps in oil paintings.

Lead white, $\mathrm{Pb}\left(\mathrm{CO}_{3}\right)_{2} \cdot \mathrm{Pb}(\mathrm{OH})_{2}$, zinc white, $\mathrm{ZnO}_{2}$, and titanium white, $\mathrm{TiO}_{2}$, are the most used white pigments in oil paintings. Both lead and zinc cations are known to be prone to react with the free fatty acids of the oil. Lead and zinc are indeed the most common metals involved in the formation of metal soaps, although metal soaps of potassium, calcium, copper, and aluminium have been also identified [5, $13,16-21]$. On the other side, titanium white does not present such reactivity since it is a photocatalytic pigment that does not behave as Lewis acid or alkali, so not able to react with the drying oil $[22,23]$.

Besides the pigments, the nature of the binder agent has been proved to influence the metal soaps formation. Although oil paintings are the works of art where the metal soap formation has been mostly studied, a few publications also report this phenomenon with beeswax, egg yolk and resins [24, 25]. In this work, two different linseed oils were used: cold-pressed linseed oil and alkali-refined linseed oil, whose concentration of free fatty acids could be different as a consequence of the extraction/refining processes. Alkali refining, indeed, reduces the amounts of free fatty acids, unlike cold pressing that does not alter the oil [26].

Different oil paintings based on lead, zinc and titanium white pigments, formulated following the traditional preparation methods, have been subjected to cyclic conditions of relative humidity (RH) and temperature. The formation of metal soaps was followed in time by ATR-FTIR, focusing on the spectral region $1650-1380 \mathrm{~cm}^{-1}$ where the stretching bands of the carboxylate groups (vCOO-) appear [27]. The results were compared with the behaviour of paintings conserved in standard environmental conditions $\left(50 \% \pm 5 \mathrm{RH}, 21 \pm 2^{\circ} \mathrm{C}\right)$.

\section{Materials And Methods}

\subsection{Sample preparation}

Titanium and zinc white pigments were purchased from Sennelier pigments manufacturer and lead (II) carbonate basic from Panreac Quimica S.A. Two types of linseed oils from Winsor\&Newton were employed: cold-pressed and alkali-refined drying oils. All the starting materials were first characterized using X-Ray Powder Diffraction (XRPD) for the pigments and Attenuated Total Reflectance FTIR spectroscopy (ATR-FTIR) for the drying oils.

Painting models were made reproducing traditional paints, by mixing the pigments with the proper amount of drying oil in order to achieve good rheology of the mixture. Table 1 shows the relative amount of each component used and the acronym given to each paint. Such paintings were applied as uniform 
layers of $120 \mu \mathrm{m}$ thickness in inert glass supports and let dry in laboratory uncontrolled conditions for 812 weeks. Figure 1 shows the prepared painting models after drying (time zero, $t_{0}$ ).

Table 1

Composition (wt\%) of the aged paints and used acronym

\begin{tabular}{|llll|}
\hline Paint & Acronym & Pigment (\%) & Oil (\%) \\
\hline Lead white + Cold-pressed linseed oil & PBCP & 96.6 & 3.4 \\
\hline Lead white + Alkali-refined linseed oil & PBAR & 96.6 & 3.4 \\
\hline Zinc white + Cold-pressed linseed oil & ZNCP & 92.3 & 7.7 \\
\hline Zinc white + Alkali-refined linseed oil & ZNAR & 92.4 & 7.6 \\
\hline Titanium white + Cold-pressed linseed oil & TICP & 91.8 & 8.2 \\
\hline Titanium white + Alkali-refined linseed oil & TIAR & 91.9 & 8.1 \\
\hline
\end{tabular}

\subsection{Ageing}

Environmental conditions with cyclic temperature $(\mathrm{T})$ and relative humidity $(\mathrm{RH})$ were tested in 8-hours cycles, for every sample. In addition, fixed parameters of $21 \pm 2{ }^{\circ} \mathrm{C}$ and $50 \pm 5 \% \mathrm{RH}$, were used as reference. To study the effect of variable temperature, samples were subjected, for 4 weeks, to cycles between $21^{\circ} \mathrm{C}$ and $50^{\circ} \mathrm{C}$ keeping $\mathrm{RH}$ fixed to $50 \%$. The variable $\mathrm{RH}$ tests were performed in two sets: low $\mathrm{RH}$ (between 30 and 50\%) and high $\mathrm{RH}$ (between 50 and 90\%) keeping temperature fixed to $21^{\circ} \mathrm{C}$ in both cases, for 6 weeks. The formation of metal soaps was weekly tracked by means of ATR-FTIR spectroscopy. As references, samples of pigments and both kinds of linseed oil ALONE, were subjected to the same ageing conditions.

\subsection{Instrumentation}

Controlled ageing was carried out in two different climatic chambers: a Vötsch VCL for temperature and low humidity tests and a Vötsch Heraeus HC 2020 for the high humidity tests.

XRPD patterns were collected at room temperature using a Bragg-Brentano geometry in a Panalytical $\mathrm{X}$ 'Pert MPD instrument using Cu radiation $45 \mathrm{kV}, 40 \mathrm{~mA}$, in an angular range of $5-90^{\circ}(2 \theta)$, step size of $0.01^{\circ}$ and $1 s$ counting time. Phase identification was conducted using the PDF-2 2002 ICDD database using the X-Pert HighScore Plus software. ATR-FTIR measurements were carried out on the external surface of the model paintings, using a Nicolet 380 spectrometer. Spectra were recorded between 4000 and $400 \mathrm{~cm}^{-1}$ after 64 scans. In order to study the progress of the metal soap formation, all spectra were normalized to the prominent $\mathrm{CH}$ stretching vibration band at $2924 \mathrm{~cm}^{-1}$ to correct for variations in the amount of sample in direct contact with the ATR crystal.

\section{Results And Discussion}




\subsection{Characterization of the raw materials}

Figure 2 shows the XRPD patterns of the commercial pigments. Lead White is made of lead carbonate (cerussite) as the main phase [PDF: 01-085-1088] and a small amount of lead carbonate hydroxide, hydrocerussite [PDF: 01-072-1144]. Zinc white is composed of zinc oxide, zincite [PDF: 00-036-1451] and titanium white of $\mathrm{TiO}_{2}$-anatase as main phase [PDF: 01-084-1286] and small amount of $\mathrm{TiO}_{2}$-rutile [PDF: 01-073-1765] and TiO [PDF: 03-065-2900].

Figure 3 displays the FTIR spectra of the unpigmented fresh cold-pressed linseed oil and alkali-refined linseed oil. No substantial differences are observed by comparing both spectra. In the $3000 \mathrm{~cm}^{-1}$ region, the stretching vibrations of $\mathrm{C}-\mathrm{H}$ bonds, due to the abundant presence of $\mathrm{CH}_{2}$ and $\mathrm{CH}_{3}$ groups in the fatty acids, are observed at $3010 \mathrm{v}(\mathrm{C}-\mathrm{H})=\mathrm{CH}[\mathrm{m}], 2958 \mathrm{v}_{\mathrm{as}}\left(\mathrm{CH}_{3}\right)$ [sh], $2924 \mathrm{v}_{\mathrm{as}}\left(\mathrm{CH}_{2}\right)[\mathrm{s}]$ and $2853 \mathrm{v}_{\mathrm{s}}\left(\mathrm{CH}_{2}\right)[\mathrm{s}]$ $\mathrm{cm}^{-1}$. The peak at $3010 \mathrm{~cm}^{-1}$ and the peak at $1656 \mathrm{~cm}^{-1}$ refers to the double bonds $\mathrm{C}=\mathrm{C}$ in the fatty acids present in fresh oil and disappear within a few days due to oxidation processes. The presence of unsaturated bonds is also responsible for the strong absorption at $721 \mathrm{~cm}^{-1}$, attributed to the bending of the (cis) $\mathrm{C}=\mathrm{C}-\mathrm{H}$ group. The intense carbonyl band of the ester linkages of triglycerides appears at 1743 $\mathrm{cm}^{-1} \mathrm{v}(\mathrm{C}=0)$ [vs]. Several bands from fatty acid and carboxylic acid functional groups are present in the region between 1600 and $600 \mathrm{~cm}^{-1}$. The peaks at $1238[\mathrm{~m}], 1160[\mathrm{~s}]$ and $1098[\mathrm{~m}] \mathrm{cm}^{-1}$ are absorption bands due to the stretching of the $\mathrm{C}-\mathrm{O}$ group in ester bonds.

\subsection{Lead white paints}

Figure 4 shows the FTIR spectra obtained from the lead white paintings (PBCP and PBAR) after four weeks of ageing under cyclic temperature conditions. In the spectra, features of lead white are indicated with asterisk and correspond to the presence of the $\mathrm{CO}_{3}{ }^{2-}$ ion as described in [28]. Bands at 2852-2954 and $1736 \mathrm{~cm}^{-1}$ are due to the siccative oil as explained before. The broad band at $3446 \mathrm{~cm}^{-1}$ due to the $\mathrm{OH}$ stretching mode, confirms a certain degree of hydrolysis of the triglycerides. The band due to the asymmetric stretching of the carboxylate groups $\left(\mathrm{v}_{\mathrm{a}} \mathrm{COO}^{-}\right)$in lead carboxylates has been reported to appear as a broad band between 1510 y $1550 \mathrm{~cm}^{-1}[9,13]$ and, as can be observed, no evidence of metal soaps formation exists after four weeks, with any of the linseed oils used. Similar results to those above mentioned were obtained in the FTIR spectra of the lead white paints stored at standard conditions, in which no metal soaps bands are visible even after six weeks.

On the other hand, a small band occurs at about $1544-1548 \mathrm{~cm}^{-1}$ attributable to lead carboxylates, after six weeks of ageing at a low cyclic relative humidity (30-50\%) (see Fig. 5). Although the signal intensity of the band is quite weak, and no other characteristic bands can help to identify the type of lead soaps, its wavelength matches a band assigned to lead stearate and palmitate in previous studies [19].

The use of cold-pressed or alkali-refined linseed oil does not suppose any difference for the reaction time of the lead white to form metal soaps, and the respective FTIR bands of the lead soaps do not show differences in intensity. However, the bands are poorly defined and longer ageing times could help to 
understand possible diversity in the behaviour of the oils. The formation of lead soaps takes place only at low relative humidity values. When the lead paintings are subjected to high relative humidity cycles or constant conditions at $50 \% \mathrm{RH}$, the band at $1544-1548 \mathrm{~cm}^{-1}$ is not formed even after six weeks (see Fig. $6)$.

Unexpectedly, at these ageing times, the low relative humidity range favours the formation of lead carboxylates over the high relative humidity conditions. However, several studies have shown how increasing values of relative humidity speed up the formation of carboxylates as a consequence of hydrolysis phenomena of drying oil $[9,13,29]$. As hydrolysis proceeds, an FTIR band at about $1710 \mathrm{~cm}^{-1}$, attributed to the carbonyl stretching $\mathrm{v}(\mathrm{C}=0)$ of the free fatty acids, increases, while the carbonyl stretching band of the ester groups decreases $[11,19,22]$. In the studied lead white paints, the band of the free fatty acids cannot be distinguished because it is hidden by the stretching vibration band of the ester groups at $1735 \mathrm{~cm}^{-1}$. Nonetheless, no decreasing trend of this latter band is appreciable over time. Moreover, the hydroxyl stretching band $\mathrm{v}(\mathrm{OH})$ at $3452 \mathrm{~cm}^{-1}$, which usually increases as the hydrolysis proceeds, does not show substantial growths over time under all ageing conditions. Therefore, no hydrolysis phenomena seem to have been favoured by any of the ageing conditions tested, and the formation of metal soaps can be attributed to the reaction of lead ions with free fatty acids already present in the oil composition and, to a lesser degree, with those released during secondary reactions of oil oxidation. For this reason, when no hydrolysis phenomena are caused, the rate of saponification strongly depends on the mobility of free fatty acids and metal ions. In the case of the aged lead white paints, the cyclic variations between low values of relative humidity could have favoured such mobility more than the other ageing conditions. However, the behaviour in longer times cannot be discussed.

\subsection{Zinc white paints}

Figure 7 shows the FTIR spectra obtained at different times during the cyclic temperature ageing of the zinc white paints, produced with cold-pressed linseed oil (ZNPC) or alkali-refined linseed oil (ZNAR), together with the reference samples kept under standard conditions $\left(21 \pm 2^{\circ} \mathrm{C}, 50 \pm 5 \% \mathrm{RH}\right)$. In both cases, a broad band at $1588 \mathrm{~cm}^{-1}$ is seen at $t_{0}$ which strongly increases in intensity after two weeks. From week 2 to week 4, no significant change occurs. This behaviour is similar in standard conditions and under cyclic temperature, no matter the linseed oil used. FTIR spectra of zinc carboxylates previously detected in paintings, show a broad intense band between 1570 y $1590 \mathrm{~cm}^{-1}$ associated with the asymmetric stretching of the carboxylate groups $\left(\mathrm{v}_{\mathrm{a}} \mathrm{COO}^{-}\right)$, whose intensity increases with the saponification reaction $[9,14,15,17]$. These observations allow us to assign the band at $1588 \mathrm{~cm}^{-1}$ to the formation of zinc soaps. FTIR spectra obtained from linseed oil or pigment alone, aged under the same conditions, do not show any new peak between 1570 and $1590 \mathrm{~cm}^{-1}$, confirming that the above-mentioned band is attributable to a secondary product of the reaction between the pigment and the drying oil, as metal soaps. Such band is already present at $t_{0}$, meaning that the formation of zinc soaps started during the drying process prior to the accelerated ageing, under indoor laboratory conditions. The ageing at a cyclic temperature between 21 and $50^{\circ} \mathrm{C}$ favours the formation of zinc soaps, as the intensity of the band at 
$1588 \mathrm{~cm}^{-1}$ increases after two weeks in a higher degree than the samples kept at standard conditions (see Fig. 7). At longer times (four weeks), the intensity barely changes. However, a further increase of the band at longer times is not discarded.

Figure 8 shows the FTIR spectra obtained in the zinc white paintings (ZNCP and ZNAR) under different ageing conditions of cyclic RH and standard conditions, and the development of the band at $1588 \mathrm{~cm}^{-1}$. In all the humidity conditions tested, the FTIR spectra of the zinc white paintings show an increase of the band at $1587 \mathrm{~cm}^{-1} \mathrm{from} \mathrm{t}_{0}$ to the first three weeks. Unexpectedly, this band continue increasing at longer times only at low relative humidity values, while for higher relative humidity values (50-90\%) or constant standard environmental conditions $\left(21 \pm 2^{\circ} \mathrm{C}, 50 \pm 5 \% \mathrm{RH}\right)$, the intensity of this band is stabilized. This trend is observed for both the cold-pressed (ZNCP) and alkali-refined (ZNAR) linseed oils. However, when fluctuant conditions are used, both under low and under high $\mathrm{RH}$ values, the band at $1588 \mathrm{~cm}^{-1}$ shows a slightly higher intensity in the cold-pressed linseed oil-based paints (ZNCP) than in the refined linseed oilbased paints (ZNAR) (see Fig. 8d-8f). This observation may be related with the higher percentage of free fatty acids present in cold-pressed linseed oil than in alkali-refined linseed oil, due to their different production processes, and consequently the greater contribution of free fatty acids in soaps formation by the former.

Crystallization of metal soaps has been reported to produce a new band at $\left(1510-1538 \mathrm{~cm}^{-1}\right)$ in the FTIR spectra, which is not observable in the samples studied, probably due to the short ageing period used [30].

As in the case of the lead white paints, an evaluation of hydrolysis of the zinc white paints is not possible at the ageing conditions. The absorption of the free fatty acids at about $1710 \mathrm{~cm}^{-1}$ is hidden by the wide ester group band at $1738 \mathrm{~cm}^{-1}$, and the latter does not show a decreasing trend as the band of the carboxylates increases in intensity. However, an appreciable grown of the hydroxyl band at about 3400 $\mathrm{cm}^{-1}$ is observed in the FTIR spectra of the paints aged with cyclic variations of relative humidity, both at high values and at low values. The hydroxyl bands are more pronounced especially when the ageing was carried out with cyclic values of low relative humidity, confirming once again the influence of these conditions on the ageing processes of the oil paints. For this reason, no phenomena of hydrolysis are excluded at all under cyclic humidity conditions.

In the other environmental conditions, spectral changes attributable to hydrolysis phenomena are not observed and, consequently, the formation of metal soaps can be attributed to the reaction of zinc ions with free fatty acids already present and, to a lesser extent, with those released by secondary oxidation processes of the oil. The stabilization of the bands of the carboxylates, observed after three weeks of ageing, could therefore depend on the availability of free fatty acids. When all the available free fatty acids have reacted and no more fatty acids are reachable by metal ions, or leachable by hydrolysis phenomena, metal soaps stop forming. At low cyclic values of relative humidity, the band keeps increasing after the third week, probably due to its capacity to favour the mobility of the reagents, as 
observed before in the lead white paints. Moreover, as mentioned above, hydrolysis phenomena are not discarded and could have helped to form metal soaps in this specific case.

\subsection{Titanium white paints}

Titanium white paintings do not show any evidence of metal soaps formation at any of the environmental conditions tested. Their FTIR spectra do not show indeed any difference in the region of the stretching vibrations of the carboxylates groups $\left(1650-1380 \mathrm{~cm}^{-1}\right)$ [27]. Figure 9 shows, as an example, the FTIR spectra of titanium paintings aged at low cycling RH. Similar behaviour is observed in the high $\mathrm{RH}$, cyclic temperature and standard environmental conditions. These observations confirm the scant propensity of this pigment to form metal soaps, already observed in previous studies [22, 23].

\section{Conclusions}

The formation of metal soaps in oil paintings has been studied by ageing model samples in controlled cyclic conditions of temperature and relative humidity. Three different white pigments (lead, zinc and titanium white) and two types of linseed oils with different production process (cold-pressed and alkalirefined) have been tested. The zinc white-based paintings formed zinc soaps under all tested conditions, both under fluctuations in temperature or humidity, and under standard conditions. However, in the case of the lead white-based paints, the formation of lead carboxylates only occurs, in the studied period, at low cyclic values of relative humidity (30-50\%). Zinc white-based paintings have, therefore, a greater propensity to form metal soaps than lead white-based paintings. However, the formation of lead soaps at longer ageing times is not discarded. Titanium white-based paintings do not form metal soaps in any of the environmental conditions, as expected, confirming that this pigment is unaffected by saponification phenomena.

During the drying period (8-12 weeks), metal soaps are already formed in the zinc paintings and continue forming during the first 3 weeks of ageing, but only under low RH (30-50\%) further increase of the carboxylate band of FTIR is observed after the sixth week of treatment.

Surprisingly, both in lead white and zinc white paintings, the formation of metal soaps was favoured especially by low cyclic values of relative humidity (30-50\%). Moreover, fluctuant values of temperature and humidity promote faster saponification than in standard conditions, confirming the importance of preserving oil paints at optimal and constant environmental conditions. Fluctuant conditions could favour the mobility of free fatty acids and metal ions, thus promoting the formation of metal soaps.

Hydrolysis phenomena were not observed, even though they are not excluded under any environmental condition. The formation of metal soaps was therefore the product of the reaction between metal ions and free fatty acids already present in oil composition or released during secondary oxidation processes. Comparing the FTIR spectra, slightly more intense bands of the carboxylates are appreciated in the coldpressed oil-based paints than in the refined linseed oil-based paints. The different processes of pressing 
or refining linseed oil can therefore modify the quantity of free fatty acids present in its composition, and consequently affect the formation of metal soaps.

\section{Abbreviations}

ATR-FTIR: Attenuated total reflectance- Fourier Transform Infrared spectroscopy.

RH: Relative Humidity

T: Temperature

$t_{0}$ : time zero of ageing, set after the drying procedure

XRPD: X-ray powder diffraction

PBCP: Lead white + cold-pressed linseed oil paint

PBAR: Lead white + alkaly-refined linseed oil paint

ZNCP: Zinc white + cold-pressed linseed oil paint

ZNAR: Zinc white + alkaly-refined linseed oil paint

TICP: Titanium white + cold-pressed linseed oil paint

TIAR: Titanium white + alkaly-refined linseed oil paint

[vs]: very strong band in FTIR spectra

[s]: strong band in FTIR spectra

[m]: medium band in FTIR spectra

[sh]: shoulder in FTIR spectra

\section{Declarations}

Availability of data and materials: The datasets generated during the current study are available from the corresponding author on reasonable request.

Competing interests: The authors declare that they have no competing interests

Funding: This research has been funded by the Complutense University and the Autonomous Community of Madrid through the project: PR65/19-22458. 
Authors contributions: Conseptualization and design: MP, MPE, MSA; Samples preparation, ageing and analysis: MP, MPE; data treatment and interpretation and manuscript writing: MP, MPE, MSA. All authors read and approved the fnal manuscript

\section{Acknowledgments}

Authors gratefully acknowledge the Complutense University for the postdoctoral position of Marta PérezEstébanez. They also thank the assistance of the research centres from Complutense University of Madrid (CAI) for the XRPD measurements.

\section{References}

1. Middelkoop N, Noble P, Wadum J, et al. Rembrandt Under the Scalpel: The Anatomy Lesson of Dr Nicolaes Tulp Dissected. Amsterdam: Six Art Promotion; 1998.

2. Noble P, Wadum J, Groen KM, et al. Aspects of 17th century binding medium: inclusions in Rembrandt's Anatomy Lesson of Dr Nicolaes Tulp. In: Art et Chimie, la Couleur: Actes du congrès, Paris, 16-18 September 1998. Paris: CNRS éd; 2000. pp. 126-9.

3. Tumosa CS. A brief history of aluminium stearate as a component of paint. WAAC Newsletter. 2001;23(3):10-1.

4. Boon JJ, Van Der Weerd J, Keune K, et al. Mechanical and chemical changes in Old Master paintings: dissolution, metal soap formation and remineralization processes in lead pigmented ground/intermediate paint layers of 17th century paintings. In: In ICOM Committee for Conservation: 13th Triennial Meeting, Rio de Janeiro, 22-27 September 2002. London: James and James (Science Publishers); 2002. pp. 401-6.

5. Centeno SA, Mahon D. The Chemistry of Aging in Oil Paintings: Metal Soaps and Visual Changes, The Metropolitan Museum of Art Bulletin. 2009; .

6. Hermans JJ. Metal soaps in oil paint: Structure, mechanisms and dynamics, PhD thesis, Van 't Hoff Institute for Molecular Sciences (HIMS), University of Amsterdam; 2017.

7. Noble P, Van Loon A, Boom JJ. Chemical changes in old master paintings II: darkening due to increased transparency as a result of metal soap formation processes. In: In ICOM Committee for Conservation: 14th Triennial Meeting, The Hague, 12-16 September 2005. London: James and James (Science Publishers); 2005. pp. 496-503.

8. Keune K, Boéve-Jones G. Its surreal: zinc-oxide degradation and misperceptions in Salvador Dalís Couple with Clouds in Their Heads, 1936. In: Van den Berg KJ, Burnstock A, editors. Issues in contemporary oil paints. Cham: Springer; 2014. pp. 283-94. doi.org/10.1007/978-3-319-10100-2_19. ., de Tagle, A., et al, editors.

9. Arbizzani R, Casellato U, Fiorin E, et al. Decay markers for the preventative conservation and maintenance of paintings. 2004; doi.org/10.1016/j.culher.2003.12.003. 
10. Erhardt D, Tumosa CS, Mecklenburg MF. Natural and accelerated thermal aging of oil paint films. Stud Conserv. 2000. doi.org/10.1179/sic.2000.45.Supplement-1.65.

11. Garrappa S, Kočí E, Švarková S, et al. Initial stages of metal soaps` formation in model paints: The role of humidity. Microchem J. 2020. doi.org/10.1016/j.microc.2020.104842.

12. Hermans JJ, Keune K, Van Loon A, et al. The crystallization of metal soaps and fatty acids in oil paint model systems. Physical Chemistry Chemical Physics. 2016. doi.org/10.1039/C6CP00487C.

13. Luxán M, Dorrego F. Reactivity of earth and synthetic pigments with linseed oil. Surf Coat Int. 1999. doi.org/10.1007/BF02720147.

14. Pratali E. Zinc oxide grounds in 19th and 20th century oil paintings and their role in picture degradation processes. Literary review, paint failure mechanisms and conditions of potential risk. CeROArt EGG 3. 2013; doi.org/10.4000/ceroart.3207.

15. Zumbühl S, Scherrer N, Ferreir E, et al. Accelerated ageing of drying oil paint - an FTIR study on the chemical alteration. Problems of accelerated ageing under variable conditions of light, temperature and relative humidity. Zeitschrift für Kunsttechnologie Konservierung. 2011. doi.org/10.24451/arbor.8067.

16. Cotte M, Checroun E, Susini J, et al. Micro-analytical study of interactions between oil and lead compounds in paintings. Appl Phys A. 2007. doi.org/10.1007/s00339-007-4213-4.

17. Hermans JJ, Keune K, Van Loon A, et al. An infrared spectroscopic study of the nature of zinc carboxylates in oil paintings. J Anal At Spectrom. 2015. doi.org/10.1039/C5JA00120J.

18. Keune K. Binding medium, pigments and metal soaps characterised and localised in paint crosssections, PhD thesis, FOM-Institute for Atomic and Molecular Physics (AMOLF), University of Amsterdam; 2005.

19. Mazzeo R, Prati S, Quaranta M, et al. Attenuated total reflection micro FTIR characterisation of pigment-binder interaction in reconstructed paint films. Anal Bioanal Chem. 2008. doi.org/10.1007/s00216-008-2126-5.

20. Plater MJ, De Silva B, Gelbrich T, et al. The characterisation of lead fatty acid soaps in "protrusions" in aged traditional oil paint, Polyhedron. 2003; doi.org/10.1016/S0277-5387(03)00461-3.

21. Robinet L, Corbeil MC. The Characterization of Metal Soaps. Studies in Conservation. 2013; doi.org/10.1179/sic.2003.48.1.23.

22. Morsch S, Van Driel BA, Van Den Berg KJ, et al. Investigating the Photocatalytic Degradation of Oil Paint using ATR-IR and AFM-IR. ACS Applied Materials Interfaces. 2017. doi.org/10.1021/acsami.7b00638.

23. Van Driel B, White, Friend or Foe? Understanding and predicting photocatalytic degradation of modern oil paintings, PhD thesis, Delft University of Technology; 2018.

24. Liang J, Scott DA. Green-Copper Containing waxy Paint On Two Egyptian Polychrome Artifacts: A Technical Study. Stud Conserv. 2014. doi.org/10.1179/2047058413Y.0000000106. 
25. Salvadó N, Butí S, Nicholson J, et al. Identification of Reaction Compounds in Micrometric Layers from Gothic Paintings using Combined SR-XRD and SR-FTIR. Talanta. 2009; doi.org/10.1016/j.talanta.2009.04.005.

26. Izzo FC. 20th century artists' oil paints: a chemical-physical survey, PhD thesis, Università Ca' Foscari Venezia; 2011.

27. Otero A, Sanches D, Montagner C, et al. Characterisation of metal carboxylates by Raman and infrared spectroscopy in works of art. J Raman Spectrosc. 2014. doi.org/10.1002/jrs.4520.

28. Miliani C, Rosi F, Daveri A, Brunetti BG. Reflection infrared spectroscopy for the non-invasive in situ study of artists' pigments. Appl Phys A. 2012. DOI 10.1007/s00339-011-6708-2.

29. Noble PA. Brief History of Metal Soaps in Paintings from a Conservation Perspective. In: Casadio F, Keune K, Noble P et al, editors. Metal Soaps in Art. Cham: Springer; 2019. pp. 1-22. doi.org/10.1007/978-3-319-90617-1_1.

30. Baij L, Hermans JJ, Keune K, et al. Time-Dependent ATR-FTIR Spectroscopic Studies on Fatty Acid Diffusion and the Formation of Metal Soaps in Oil Paint Model Systems. Angew Chem. 2018. doi.org/10.1002/ange.201712751.

\section{Figures}

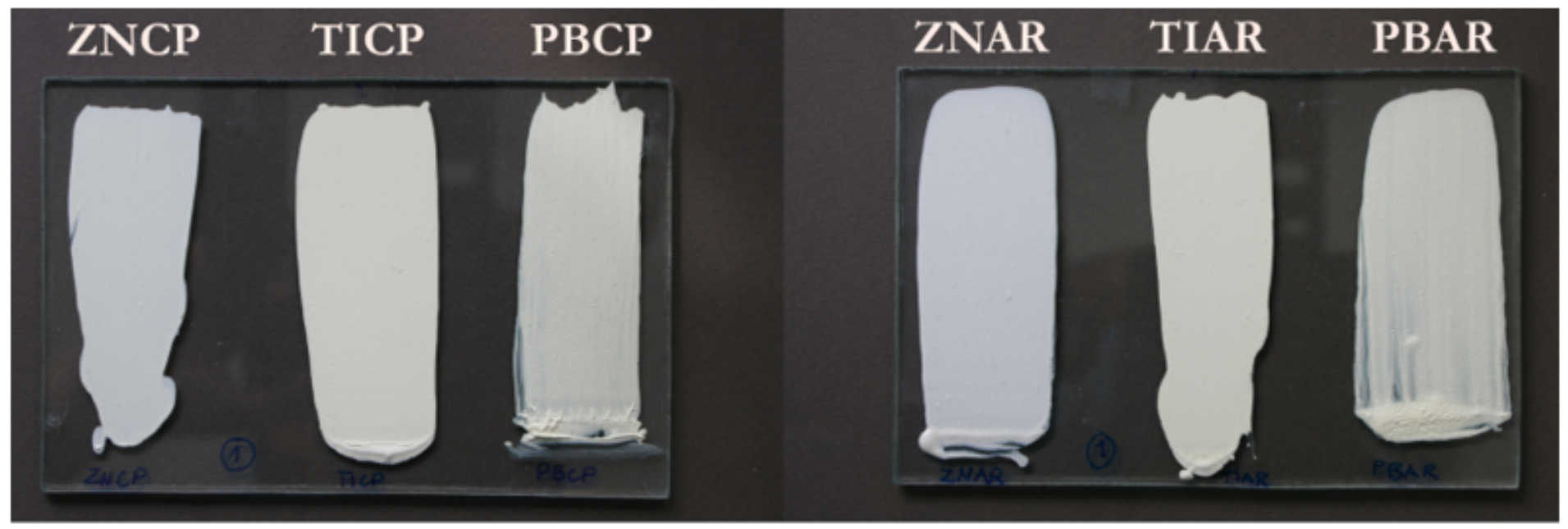

\section{Figure 1}

Painting models after drying (t0) 

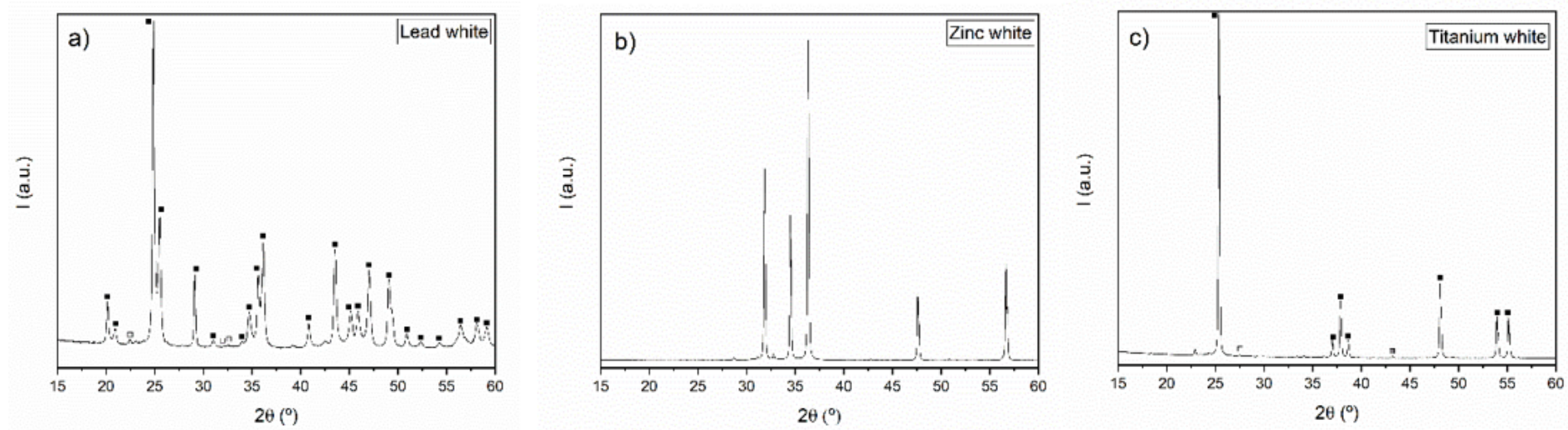

Figure 2

XRPD patterns of the used pigments: a) Lead white ( $\square \mathrm{PbCO} 3$ and $\square \mathrm{Pb3}(\mathrm{CO} 3) 2(\mathrm{OH}) 2$ ); b) zinc white and c) titanium white ( $\square$ TiO2-Anatase, $\square$ TiO2-Anatase Rutile and TiO).

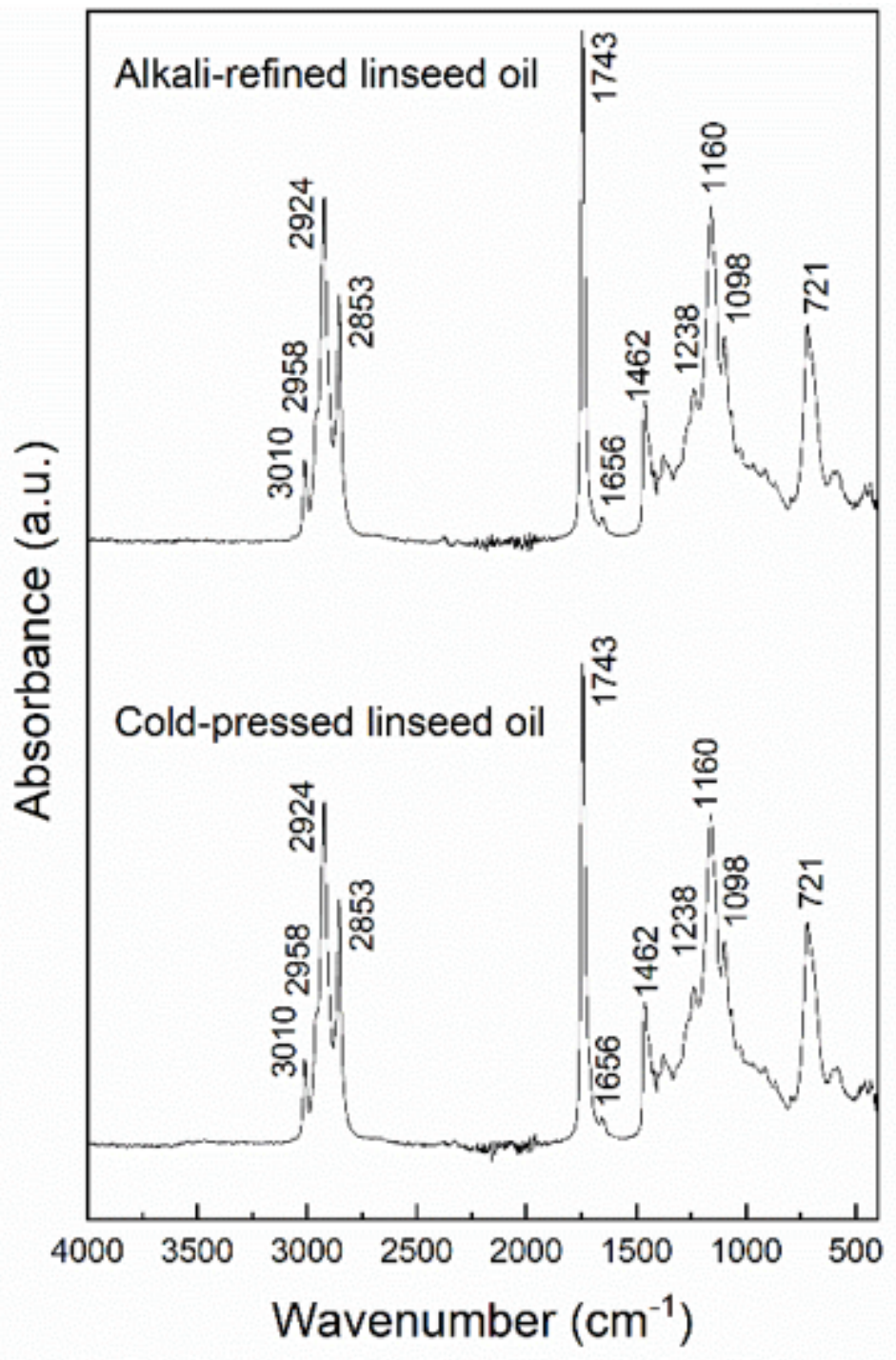

Figure 3 
FTIR spectra of the used linseed oils: cold-pressed (bottom) and alkali-refined (top).

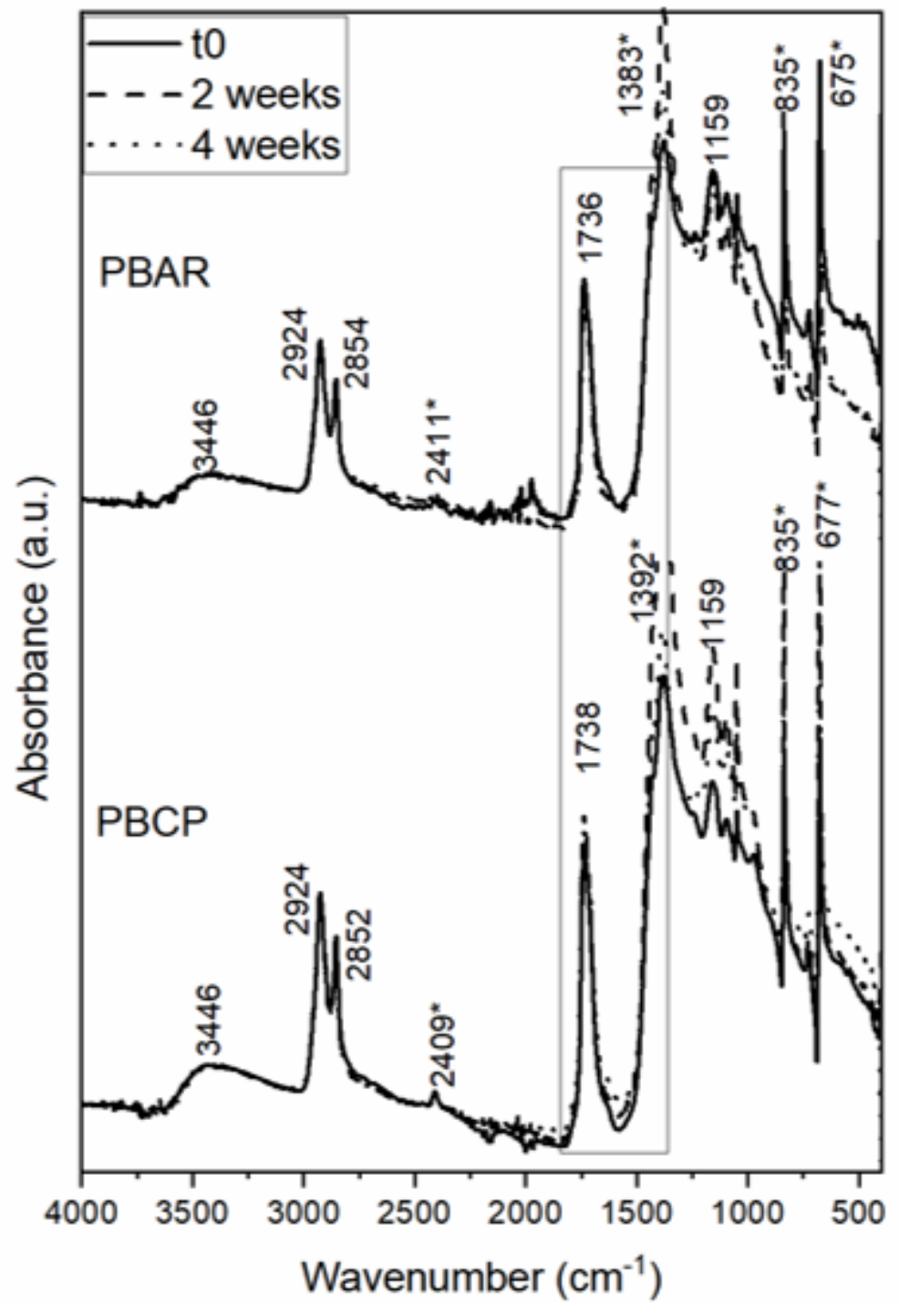

Figure 4

FTIR spectra of PBCP (bottom) and PBAR (top) aged under cyclic conditions of temperature between 21 and $50^{\circ} \mathrm{C}$, at $50 \% \mathrm{HR}$. Box indicates the area where bands due to lead carboxylates should appear. 

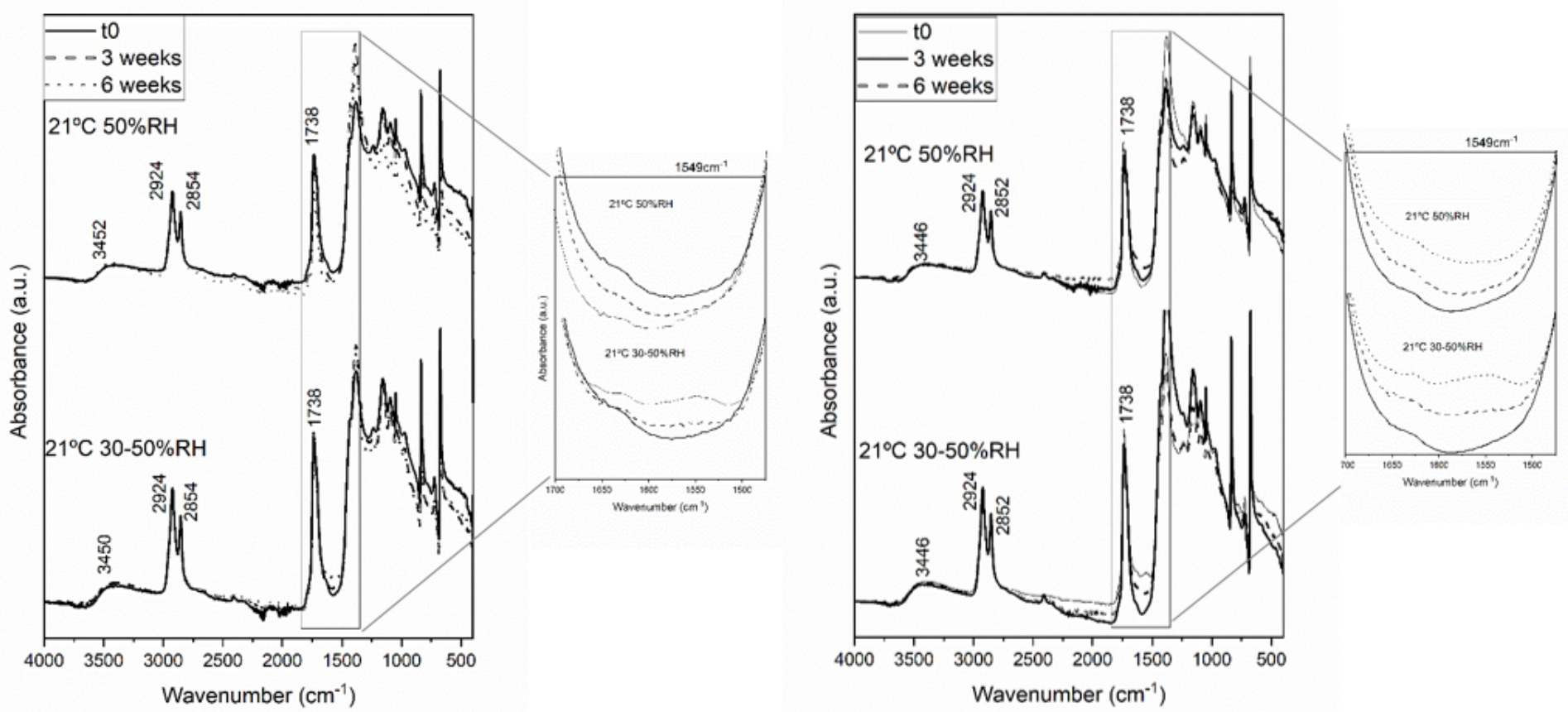

Figure 5

FTIR spectra of PBAR (bottom) and PBCP (top) aged under cyclic conditions of relative humidity between 30 and $50 \%$ and $21^{\circ} \mathrm{C}$, compared with those kept under standard conditions 


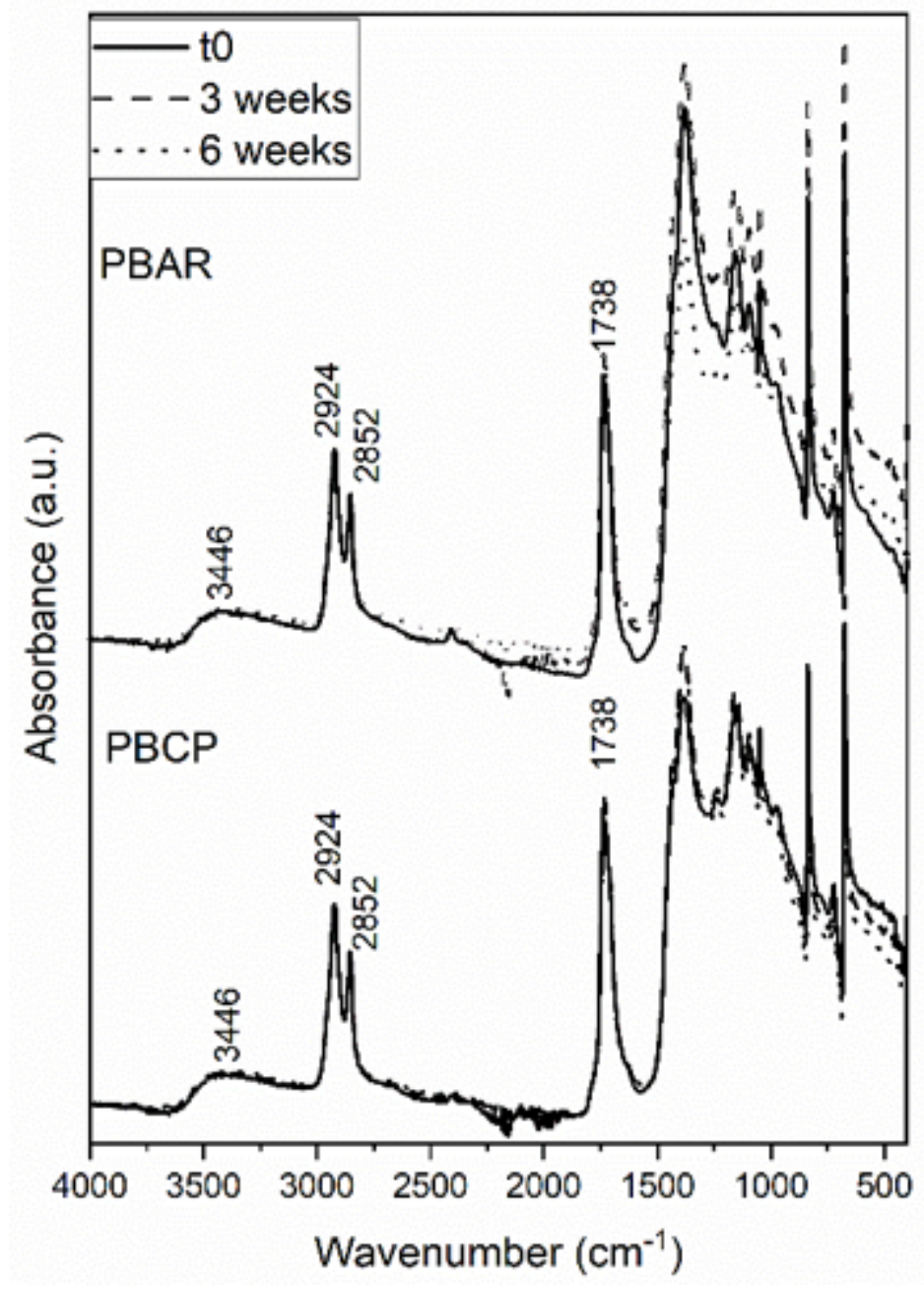

Figure 6

FTIR spectra of PBCP (bottom) and PBAR (top) aged under cyclic conditions of relative humidity between 50 and $90 \%$ and $21^{\circ} \mathrm{C}$.
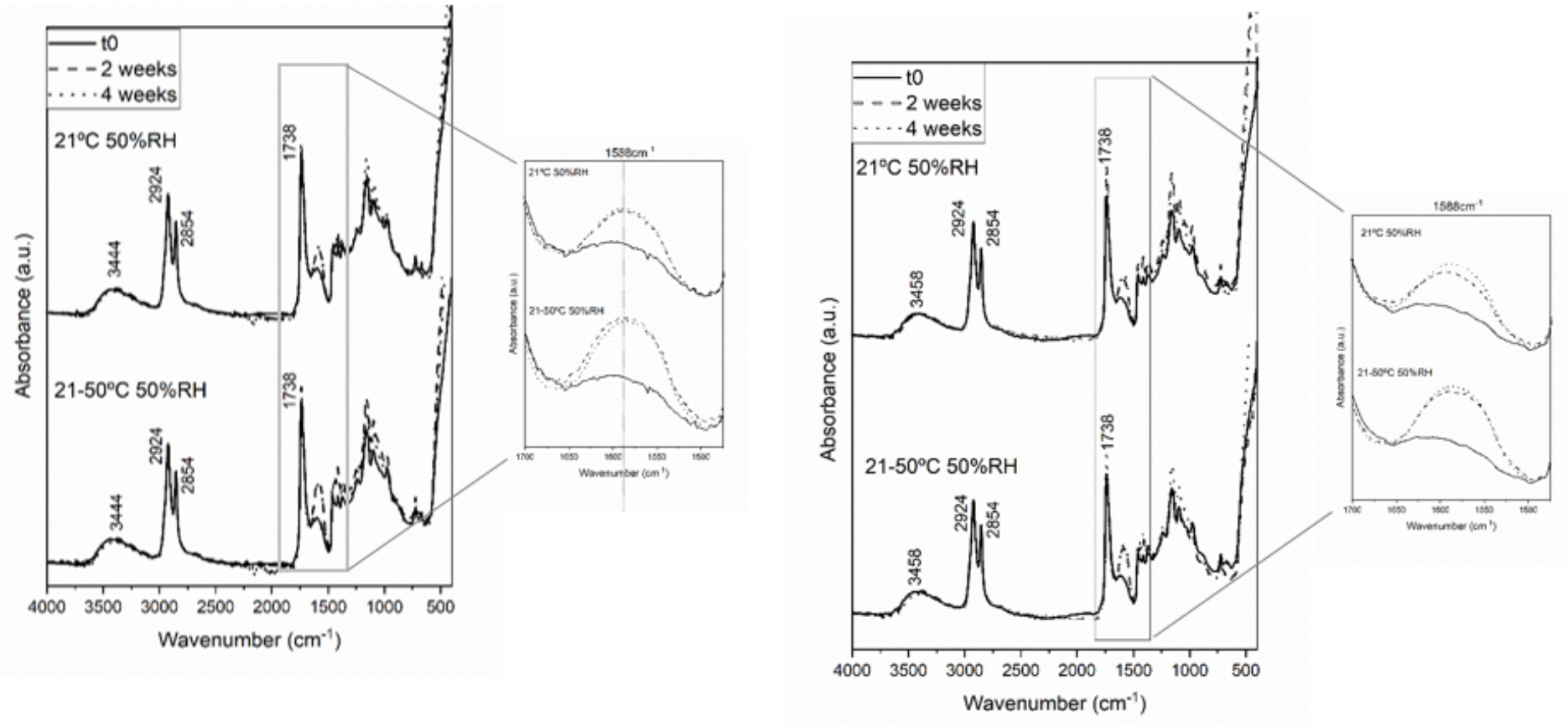
Figure 7

FTIR spectra of ZNCP (top) and ZNAR (bottom) aged under cyclic conditions of temperature between 21 and $50^{\circ} \mathrm{C}$ and $50 \% \mathrm{RH}$, compared with those kept under standard conditions
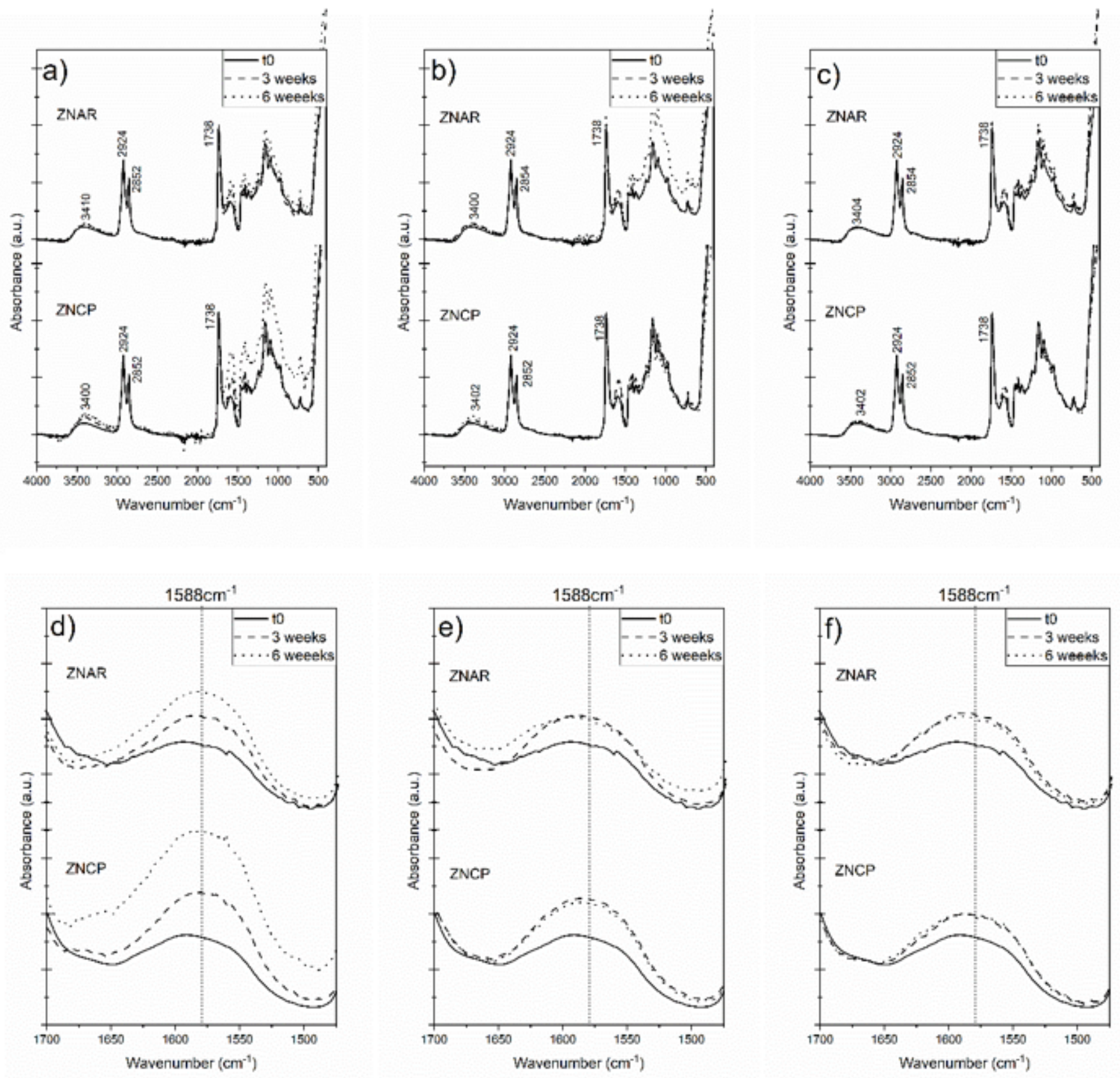

\section{Figure 8}

FTIR spectra of zinc white paintings (ZNCP and ZNAR) under different ageing conditions: $30-50 \% \mathrm{RH}$ and $21^{\circ} \mathrm{C}$ (a), $50-90 \% \mathrm{RH}$ and $21^{\circ} \mathrm{C}$ (b) and $50 \pm 5 \% \mathrm{RH}$ and $21 \pm 2^{\circ} \mathrm{C}$ (c). Development of the FTIR band at 1588 $\mathrm{cm}-1$ with ageing time at $30-50 \% \mathrm{RH}$ and $21^{\circ} \mathrm{C}(\mathrm{d}), 50-90 \% \mathrm{RH}$ and $21 \pm 2^{\circ} \mathrm{C}(\mathrm{e})$ and $50 \pm 5 \% \mathrm{RH}$ and $21 \pm 2^{\circ} \mathrm{C}$ (f) 


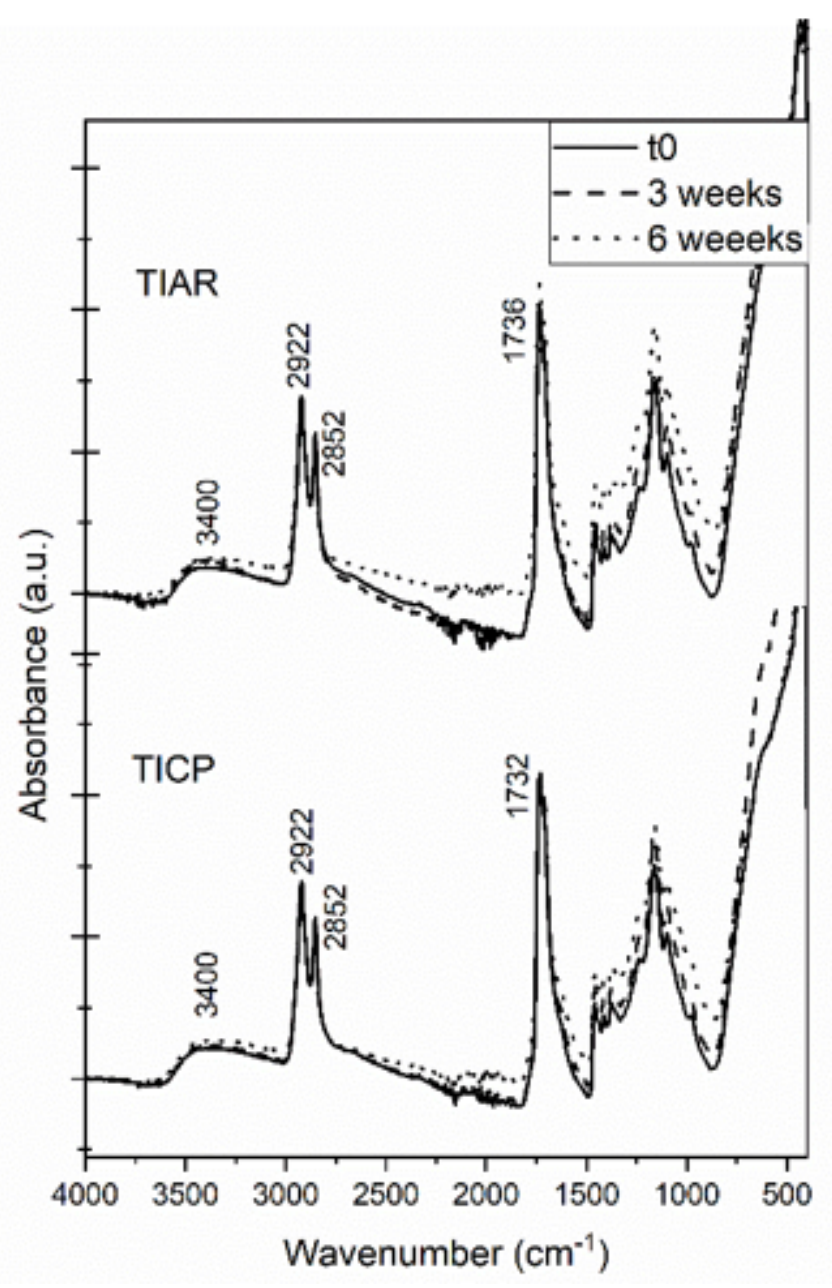

Figure 9

FTIR spectra of titanium-based paintings (TICP and TIAR) aged under cyclic conditions of relative humidity between 30 and $50 \%$ and $21^{\circ} \mathrm{C}$. 